\title{
Intrinsic thermal vibrations of suspended doubly clamped single-wall carbon nanotubes
}

\author{
B. Babić, J. Furer, S. Sahoo, Sh. Farhangfar, and C. Schönenbergel* \\ Institut für Physik, Universität Basel, Klingelbergstr. 82, CH-4056 Basel, Switzerland
}

(Dated: May 9, 2019)

\begin{abstract}
We report the observation of thermally driven mechanical vibrations of suspended doubly clamped carbon nanotubes, grown by chemical vapor deposition (CVD). Several experimental procedures are used to suspend carbon nanotubes. The vibration is observed as a blurring in images taken with a scanning electron microscope. The measured vibration amplitudes are compared with a model based on linear continuum mechanics.
\end{abstract}

PACS numbers: 61.46.+w, 62.25.+g, 62.30.+d, 81.07.De

Carbon nanotubes (NTs) form a material with unique mechanical properties [1, 2, 3, 4, 5]. The high Young's modulus and low specific weight qualify single-wall carbon nanotubes (SWNTs) as ultimate mechanical resonators. Similar to lithographically patterned $\mathrm{SiC}$ beams, whose resonance frequency has recently crossed the border from $\mathrm{MHz}$ to $\mathrm{GHz}[\underline{6}$ ], it would be highly desirable to integrate NTs into nanoelectromechanical systems (NEMSs) and to electrically excite the mechanical vibration modes [7]. A first step in this direction has been the observation of electrically driven mechanical vibrations of multi-wall carbon nanotubes [3]. Nanometersized resonators oscillate at high frequencies, but simultaneously have small vibration amplitudes, which are difficult to measure. At cryogenic temperatures, the resonant adsorption of an external electromagnetic field could successfully be measured using superconducting elements attached to a freely suspended NT [8]. At room temperature a tunnelling probe in the form of, for example, an STM tip would be a versatile detector. Integrating a sensitive measuring transducer with a NT nanomechanical oscillator is however challenging. In a first step, it would be desirable if the mechanical vibrations could be imaged directly. Here, we report on the observation of thermal vibrations of suspended doubly clamped SWNTs, imaged by scanning electron microscopy (SEM).

Thermally driven excitations of multi-wall carbon nanotubes (MWNTs), clamped at one end only, were first investigated by Treacy et al. [1]. The mechanical oscillation appeared in the images, which were collected with a transmission electron microscope (TEM), as a blurring that increased towards the free end of the MWNTs.

FIG. 1: Schematic drawings of doubly clamped vibrating SWNT which are suspended by different methods. (a) top view, (b-d) side views.

*Electronic address: Christian.Schoenenberger@unibas.ch URL: www. unibas.ch/phys-meso
In order to see whether a similar experiment is possible with doubly clamped SWNTs, we will first estimate the expected amplitude in thermal equilibrium at room temperature. A schematics with coordinate system is shown in Fig. 1a. Assuming that linear continuum mechanics is a good approximation, the equation of motion for the vertical displacement $\xi$ is given by $[\underline{9}$

$$
\frac{\partial^{2} \xi}{\partial t^{2}}+\left(\frac{Y I}{\rho A}\right) \frac{\partial^{4} \xi}{\partial x^{4}}=0
$$

Here, $\rho$ is the mass density, $A$ the cross-sectional area, $Y$ the Young's modulus, and $I=\pi d^{4} / 64$ the moment of inertia, which depends only on the diameter $d$. Applying the proper boundary conditions, the spectrum of eigenfrequencies is obtained:

$$
\omega_{i}=\frac{\beta_{i}^{2}}{L^{2}} \sqrt{\frac{Y I}{\pi d \rho_{2 d}}} \quad(i=1,2,3 \ldots),
$$

where $L$ is the suspended length, $\rho_{2 d}$ is the surface mass density of a graphite sheet $\left(7.7 \cdot 10^{-7} \mathrm{kgm}^{-2}\right)$ and $\beta_{1}=$ 4.73, $\beta_{2}=7.85$, and $\beta_{3}=11.0$ for the first three modes.

The equipartition theorem predicts that each vibration mode carries the energy $k_{B} T$ in thermal equilibrium at temperature $T$, where $k_{B}$ is the Boltzmann constant. Together with the appropriate solutions of Eq. (II), one obtains an expression for the variance of the maximum deflection amplitude, which for the fundamental frequency $(i=1)$ occurs in the middle:

$$
\sigma_{1}^{2} \equiv<\xi_{1}^{2}(L / 2)>=\frac{k_{B} T L^{3}}{\gamma_{1} Y I},
$$

where $\gamma_{1}=192$ 10]. Table [ summarizes the eigenfrequencies and the thermal vibration amplitudes at room temperature of a 'typical' SWNT with diameter $d=1.5 \mathrm{~nm}$ and Young's modulus $Y=1 \mathrm{TPa}$ for different (practically feasible) suspension lengths $L=0.2-5 \mu \mathrm{m}$. This table demonstrates that thermal vibration amplitudes can be of appreciable magnitude, of order $\approx 10 \mathrm{~nm}$ $(L=1 \mu \mathrm{m})$. Since state-of-the-art scanning electron microscopes have resolutions well below $10 \mathrm{~nm}$, thermal vibration should appear on SEM images. 
TABLE I: Characteristic quantities of suspended SWNT $(d=1.5 \mathrm{~nm}, Y=1 \mathrm{TPa})$. The eigenfrequencies and maximum thermal amplitudes are calculated using relations (2) and (3), respectively.

\begin{tabular}{ccc}
$L(\mu \mathrm{m})$ & $\omega(\mathrm{MHz})$ & $\sigma_{1}(\mathrm{~nm})$ \\
\hline \hline 0.2 & 4600 & 0.8 \\
0.5 & 740 & 3.4 \\
1 & 185 & 9.3 \\
3 & 21 & 48.3 \\
5 & 7.4 & 104 \\
\hline
\end{tabular}

There are already many reports on the fabrication of suspended NTs. For example, SWNTs were grown between distant silicon towers 11, 12], spread over metal posts 13], or grown over solid terraces 14] and etched trenches [4]. Though devices with suspension lengths of $L \gtrsim 5 \mu \mathrm{m}$ were realized and imaged with SEM, the thermal vibration has surprisingly not yet been reported, although it should readily have shown up in respective SEM images, provided the reported SWNTs were single SWNTs. In the work of Dai and coworkers 11, 12, 14], the SWNTs were coated with a metal layer to increase the contrast in the SEM, whereas others have explicitly reported on suspended ropes of SWNTs or MWNT [5, 13], which are inherently stiffer.

Carbon nanotubes are synthesized by chemical vapor deposition (CVD) as previously reported 15]. We would like to emphasize that not all grown NTs are individual SWNTs. This will be explained further in the text. To account for the possible influence of substrate during imaging in SEM, we have suspended NTs using three different methods.

Method I, shown in Fig. 1b, is based on the work of Nygård et al. [16]. The NTs are grown on thermally oxidized $(400 \mathrm{~nm}) \mathrm{Si}$ substrates. Electrical contacts are patterned by electron-beam lithography (EBL), followed by evaporation $(\mathrm{Ti} / \mathrm{Au})$ and lift-off. The $\mathrm{SiO}_{2}$ is etched in buffered HF [17]. To stop etching, the sample is heavily rinsed in water followed by isopropanol. With this method we find it possible to suspend NTs over distances up to $1 \mu \mathrm{m}$. For larger lengths, the surface tension of the etchant tends to pull the NT down to the substrate.

In method II, shown in Fig. 1c, the NTs are grown across predefined trenches. We start with a Si substrate with layers of $800 \mathrm{~nm}$ of $\mathrm{SiO}_{2}$ and $200 \mathrm{~nm}$ of $\mathrm{Si}_{3} \mathrm{~N}_{4}$. Slits of width $1-5 \mu \mathrm{m}$ and length $10 \mu \mathrm{m}$ are first etched into the top $\mathrm{Si}_{3} \mathrm{~N}_{4}$ layer using a $\mathrm{CHF}_{3}$-based plasma etching process [19]. Next, the slit is further wet-etched into $\mathrm{SiO}_{2}$ and the Si substrate using $\mathrm{HF}$ and $\mathrm{KOH}$ [20], respectively. This results in deep trenches $\sim 3.5 \mu \mathrm{m}$, a prerequisite for NTs to bridge the trenches in the CVD growth process.

In method III, shown in Fig. 1d, slits are defined in $\mathrm{Si}_{3} \mathrm{~N}_{4}$ membranes of thickness $150 \mathrm{~nm}$ and lateral size $0.5 \mathrm{~mm}$ following a similar procedure as in method II.

The key difference between the three methods is the depth of suspension. It is $400 \mathrm{~nm}, 3.5 \mu \mathrm{m}$, and $\infty$ for
FIG. 2: (a) SEM image of a vibrating SWNT grown over a trench. A strong blurring is clearly visible (indicated by arrows), which is a consequence of intrinsic thermal vibrations. (b) Another vibrating NT, whose root-mean-square displacement along $x$ is plotted in (c). Circles are measured points and the curves represent fits.

FIG. 3: (a) SEM image of long $(L \approx 6.2 \mathrm{~nm})$ vibrating SWNT grown over a slit in a $\mathrm{Si}_{3} \mathrm{~N}_{4}$ membrane. In (b) three NTs are imaged simultaneously. Only the middle one is vibrating. A white circle indicates branching of the lower NT into two NTs.

methods I-III, respectively. The samples are imaged with SEM (Philips XL30 FEG) at room temperature. To generate an image, a focused electron beam is raster scanned.

To deduce the vibration amplitude quantitatively two assumptions have to be made: 1) the intensity profile of the electron beam centered at coordinate $(x, y)$ has a Gaussian distribution and 2) the measured intensity of secondary electrons reflects the (time-averaged) probability $P(x, y) \equiv P_{x}(\xi)$ to find the NT at position $(x, y)$ convoluted with the intensity profile of the primary beam. 1) is a convenient assumption and 2) should hold, because scanning in SEM is slow as compared to the vibration of the NT. The latter results in a blurring of the NT in SEM images. An example of a vibrating suspended NT is shown in Fig. 2a. The vibration is observed as a blurring, which is largest in the middle. In contrast, the NT appears sharp at the edges of the trench, limited by the finite resolution of the SEM. To deduce the vibration amplitude, more precisely the variance $\sigma^{2}(x) \equiv\left\langle\xi^{2}(x)\right\rangle$, we note that $P_{x}(\xi)$ is Gaussian and determined by Boltzmann statistics. The deconvolution is simple because of assumption 1$)$. We only need to extract $\sigma^{2}(x)$ from the intensity distribution of the SEM image perpendicular to the NT and subtract $\sigma^{2}(0)$. To do so, we average the intensity profile in $\Delta x$ slices as shown in Fig. $2 \mathrm{~b}$ and fit it to a Gaussian. Such an analysis was first done for MWNT cantilevers by Krishnan et al. 18].

Figure $2 \mathrm{~b}$ shows a SEM image of a suspended doubly clamped vibrating NT fabricated by method I. The free suspension length is relatively short, i.e. $L \approx 650 \mathrm{~nm}$. Applying the analysis procedure mentioned above, the maximum rms vibration amplitude is determined to be $\sigma=27 \pm 5 \mathrm{~nm}$. We have also analyzed $\sigma$ as a function of $x$ and compare the result with analytical curves for the first three eigenmodes in Fig. 2c. The agreement between the measured points and the theoretical curves is reasonably good. Matching between experiment and theory is improved if the first and second modes are taken into account, each of which carries $k T$ energy. Contributions from higher order modes decay very rapidly and can be neglected. Note, there is one fitting parameter $Y d^{4}$, which will be discussed below. 
Figure 3a shows another NT grown over $\mathrm{Si}_{3} \mathrm{~N}_{4}$ membrane. Here, the suspension length is rather large, i.e. $L \approx 6.2 \mu \mathrm{m}$. Correspondingly, the observed blurring is much larger. The maximum rms vibration amounts to $\sigma=80 \pm 5 \mathrm{~nm}$. Applying Eq. (3) and assuming the typical high Young's modulus value of SWNTs of $Y=1 \mathrm{TPa}$ the diameter of this $\mathrm{NT}$ is estimated to be $d=2 \pm 0.5 \mathrm{~nm}$.

The SEM image displayed in Fig. 3b shows three suspended NTs. Though grown in one run, only one NT seems to vibrate, namely the middle one. This, at first sight surprising result, points to a variability of NTs that are grown during one and the same process. The only parameter in our experiment, which is not predetermined, is $Y d^{4}$, see Eq. (3). Though different values for the Young's modulus were reported, we suspect that the diameter $d$ is the cause for the variability, because it enters in the fourth power. The absence of visible vibrations for the upper and lower NT in Fig. 3b suggests that these have a larger diameter. They may be multi-wall nanotubes or ropes of tubes. In fact, the lower one must be a rope, because a clear branching is observed at the right end (highlighted by a circle). Having looked through a large number of samples, the fraction of vibrating tubes is very small (a few \%). This is a clear indication that not all of the grown NTs are SWNTs.

TABLE II: Properties of some vibrating NTs. $L$ is the suspended length, $\sigma$ the measured maximum rms vibration, $Y d^{4}$ obtained using Eq. $3 Y_{1.6}$ Youngs' modulus assuming $d=1.6 \mathrm{~nm}$ (see text), and $d_{1}$ the NT diameter assuming $Y=1 \mathrm{TPa}$.

\begin{tabular}{cccccc}
$\begin{array}{c}L \\
(\mu \mathrm{m})\end{array}$ & $\begin{array}{c}\sigma \\
(\mathrm{nm})\end{array}$ & $\begin{array}{c}Y d^{4} \\
\left(\mathrm{GPa}(\mathrm{nm})^{4}\right)\end{array}$ & $\begin{array}{c}Y_{1.6} \\
(\mathrm{GPa})\end{array}$ & $\begin{array}{c}d_{1} \\
(\mathrm{~nm})\end{array}$ & Method \\
\hline \hline 0.55 & 25 & 117 & 18 & 0.58 & $\mathrm{I}$ \\
0.63 & 27 & 150 & 23 & 0.62 & $\mathrm{I}$ \\
1.35 & 16 & 4221 & 644 & 1.4 & $\mathrm{III}$ \\
4.05 & 85 & 4038 & 616 & 1.4 & $\mathrm{II}$ \\
4.30 & 90 & 4311 & 658 & 1.45 & II \\
6.25 & 80 & 16754 & 2556 & 2.0 & III \\
\hline
\end{tabular}

We summarize the measured rms vibration of several NTs in table [I Determined are $L$ and $\sigma(L / 2)$. Using Eq. (3), we obtain an estimate for $Y d^{4}$, which is given in the third column. What is immediately noticed is the large spread in $Y d^{4}$ of more than two orders of magnitude. Unfortunately, we are not able to unambiguously deduce the Young's modulus $Y$ and diameter $d$, independently. We have tried to measure the diameter using atomic-force microscopy (AFM). Due to surface roughness and the strong $d^{4}$ dependence, the error bar is too large to deduce $Y$ with an acceptable accuracy. For the discussion we instead rely on an average diameter for SWNTs, which we have obtained from electrical measurements of contacted semiconducting NTs [15]. We have analyzed the band-gap, which is inversely proportional to the diameter $d$, of more than 10 semiconducting SWNTs and obtained as an average $d=1.6 \pm 0.3 \mathrm{~nm}$. We note, that taking this diameter, the estimated Young's modulus (column 4 in table III denoted by $Y_{1.6}$ ) has an accuracy of 'only' $75 \%$. Well graphitized NTs have a large Young's modulus. For example, $Y=1.4 \pm 0.4 \mathrm{TPa}$ was reported for SWNTs grown by laser ablation [18], whereas $1 \mathrm{TPa}$ was found in simulations independent of helicity and number of shells 21]. In column 5 of table [II we therefore also list the diameter $d_{1}$, which we deduce from the measured $Y d^{4}$, assuming $Y=1 \mathrm{TPa} . d_{1}$ is varying between 0.58 to $2.0 \mathrm{~nm}$. Since we have never observed SWNTs with diameters $<1 \mathrm{~nm}$ in TEM, the first two NTs (row 1 and 2), both belonging to samples prepared by method I, cannot have a large Young's modulus $Y \sim 1 \mathrm{TPa}$. Taking $d$ to be $1.6 \mathrm{~nm}$ leads to a modulus of only $Y_{1.6} \approx 20 \mathrm{GPa}$. Because method I uses HF-etching we suspect that the NT's are affected during this process step. It is possible that CVD-grown SWNTs are not perfect so that wet etching can proceed starting at defect sites. In contrast to method I, the as-grown CVD NTs of methods II and III yield consistent results, which are in agreement with a large Young's modulus of $1 \mathrm{TPa}$ and with the diameter, which we have deduced by electrical measurements. Though we observe ropes and small diameter MWNTs (only a few number of shells) in TEM, their diameter is typically larger than $2 \mathrm{~nm}$. This strongly suggests that the NTs of row $3-6$ in table III are single-wall carbon nanotubes.

To our knowledge there are no reports on the Young's modulus of CVD-grown SWNT. Though we are not able to accurately determine $Y$, our results suggest that CVD-grown SWNTs can have a large modulus of order $Y \approx 1 \mathrm{TPa}$. The exception are wet-etched NTs, for which our data suggest $Y \ll 1 \mathrm{TPa}$. Small Young's modulus have previously been reported for CVD-grown MWNTs 22.

In conclusion, we have demonstrated that it is possible to observe thermally driven vibrations of suspended doubly clamped SWNTs in SEM. From the measured rms vibration amplitude, the Young's modulus $Y$ of CVDgrown SWNTs has been estimated. Only a small fraction of suspended NTs are seen to vibrate, although they are suspended over a comparable length and grown at the same time. This suggests that the majority of grown tubes are not single SWNTs, but rather ropes and MWNTs, a finding, which is supported by TEM. We suspect that this is the reason why thermal vibrations of SWNTs has not already been observed before.

\section{Acknowledgments}

We acknowledge support and technical assistance with TEM imaging by A. Engel, G. Gantenbein and H. Stahlberg of the Biocenter and discussions with L. Forró. This work has been supported by COST (BBW), the 
Swiss NFS and the NCCR on Nanoscience.

[1] Treacy, M. M. J.; Ebbesen, T. W.; Gibson, J. M. Nature 1996, 381, 678 .

[2] Lourie, O.; Wagner, H. D. Journal of Materials Research 1998, 13(9), 2418.

[3] Poncharal, P.; Wang, Z. L.; Ugarte D.; Heer, W. A. Science 1999, 283, 1513.

[4] Walters, D. A.; Ericson, L. M.; Casavant, M. J.;Liu J.; Colbert, D. T.; Smith, K. A. Smalley, R. E. Appl. Phys. Lett. 1999, 74, 3803.

[5] Yu, M. F.; Lourie, O.; Dyer, M. J.; Moloni, K.; Kelly T. F.; Ruoff, R. S. Science 2000, 287, 637.

[6] Ming, X.; Huang, H.; Zorman, C. A.; Mehregany, M.; Roukes, M. L. Nature 2003, 421, 496.

[7] Roukes, M. L. Physics World 2001, 14, 25.

[8] Reulet, B.; Kasumov, A. Yu.; Kociak, M.; Deblock, R.; Khodos, I. I.; Gorbatov, Yu. B.; Volkov, V. T.; Journet, C.; Bouchiat H. Phys. Rev. Lett. 2000, 85, 2829.

[9] See, e.g., Landau L. D.; Lifschitz E. M. Theory of Elasticity; Permagon Press, 1959.

[10] Sapmaz, S.; Blanter, Ya. M.; Gurevich L.; van der Zant, H. S. J. Phys. Rev. B 2003, 67, 235414.

[11] Cassell, A. M.; Franklin, N. R.; Tombler, T. W.; Chan, E. M.; Han, J.; Dai, H. J. Am. Chem. Soc. 1999, 121, 7975 .
[12] Franklin, N. R.; Dai, H. Adv. Mater. 2000, 12, 890.

[13] Kim, G.; Gu, G.; Waizmann, U.; Roth, S. Appl. Phys. Lett. 2002, 80, 1815.

[14] Franklin, N. R.; Wang, Q., Tombler, T. W.; Javey, A.; Shim, M.; Dai, H. Appl. Phys. Lett. 2002, 81, 913.

[15] Babić, B.; Iqbal, M.; Schönenberger, C. Nanotechnology 2003, 14, 327.

[16] Nygård, J.; Cobden, D. H. Appl. Phys. Lett. 2001, 79, 4216.

[17] Buffered hydrofluoric acid is made according to the following recepie: $28 \mathrm{ml} \mathrm{HF}+107 \mathrm{ml} \mathrm{H}_{2} \mathrm{O}+113 \mathrm{~g} \mathrm{NH} \mathrm{N}_{4} \mathrm{~F}$. The etch rate for $\mathrm{SiO}_{2}$ is $50 \mathrm{~nm} / \mathrm{min}$.

[18] Krishnan, A.; Dujardin, E.; Ebbesen, T. W.; Yianilos, P. N.; Treacy, M. M. J. Phys. Rev. B 1998, 58, 14013.

[19] Tilman Hoss, PhD thesis, University of Basel, pp 22, 2000.

[20] The etch ratio of $\mathrm{Si}$ to $\mathrm{SiO}_{2}$ is $\approx 500$, for $20 \%$ weight mass of $\mathrm{KOH}$ at $60{ }^{\circ} \mathrm{C}$.

[21] Lu, J. P. Phys. Rev. Lett. 1997, 79, 1297.

[22] Salvetat, J-P.; Kulik, A. J.; Bonard, J-M.; Andrew, G.; Briggs, D.; Stöckli, T.; Méténier, K.; Bonnamy, S.; Béguin, F.; Burnham, N. A.; Forró L. Adv. Mater. 1999, $11,161$. 